\title{
Sensitivity of Fever Sentinel Surveillance System to Survey Malaria Trends in Madagascar, 2014-2015
}

Léa Randriamampionona ${ }^{1}$, Laurence Randrianasolo ${ }^{2 *}$, Rindra Vatosoa Randremanana ${ }^{2}$, Charles Ramarokoto ${ }^{2}$, Toky Ramarokoto ${ }^{2}$, Annett Cotte ${ }^{3}$, Arsène Ratsimbasoa ${ }^{1}$, Maherisoa Ratsitorahina ${ }^{1}$, Judith Hedge ${ }^{3}$ and Patrice Piola ${ }^{2}$

${ }^{1}$ Ministry of Public Health, Madagascar

${ }^{2}$ Epidemiology and clinical Reseacrh Unit, Institut Pasteur de Mdagascar, Madagascar

3US Centers for Disease Control and Prevention, US

"Corresponding author: Laurence Randrianasolo, Epidemiology and Clinical Research Unit, Institute Pasteur de Madagascar, Antananarivo, Madagascar, Post Box: 1274101, Antananarivo, Madagascar, Phone: (261)202241274; E-mail: laurence@pasteur.mg

Received date: July 16, 2018; Accepted date: September 18, 2018; Published date: September 30, 2018

Copyright: (C) 2018 Randriamampionona L, et al. This is an open-access article distributed under the terms of the Creative Commons Attribution License; which permits unrestricted use; distribution; and reproduction in any medium; provided the original author and source are credited.

\begin{abstract}
Fever sentinel surveillance system involving 34 health centers was set up in Madagascar to detect epidemicprone diseases in real time. Evaluative research was performed to assess the proportion of febrile cases that are not captured by sentinel sites. Capture-recapture method was used with two independent data sources: the first source was a passive detection of fevers in health centers while the second was an active screening of febrile subjects in the catchment area of each corresponding health center. Cases common to both sources were identified by matching name, age and location. Completeness of collected data was estimated through the population census. In 2014-15, six health centers were randomly selected from the sentinel network to perform the study. Active screening in the catchment areas of the six health centers detected 2,902 febrile illnesses among 149,835 inhabitants. Acute malaria represented $0.3 \%(10 / 2902)$ of febrile illnesses. The passive screening in health centers notified 157 cases of fever of which $7.6 \%(12 / 157)$ were acute malaria. The estimated number of febrile cases and acute malaria in the catchment areas based on the capture recapture analysis of data from the health facilities and the active screening was 3,829 [95\% Cl: 3,498-4,160] and 17 [95\% Cl: 7-27] respectively. The overall sensitivity of sentinel health centers to detect febrile illnesses and acute malaria was $4.1 \%$ and $70.0 \%$ respectively. Therefore most malaria cases were captured by the sentinel fever surveillance system. These results will serve as a baseline for future evaluative research of the fever sentinel surveillance system in Madagascar.
\end{abstract}

Keywords: Malaria; Madagascar; Evaluation; Capture-recapture; Fever sentinel surveillance

\section{Introduction}

Communicable diseases, particularly epidemic-prone diseases, remain a major cause of morbidity and mortality in developing countries including Madagascar [1]. Most infectious diseases can be prevented and/or treated. Therefore responses to epidemics involve preventive measures and case management with greater chances of success when outbreaks are quickly notified. Abnormal epidemiological situations, or alerts, usually result from a communicable disease surveillance system with sufficient sensitivity (detect all abnormal signals) and specificity (detect only abnormal signals). A sensitive surveillance system implies the detection and reporting of the specific diseases under surveillance.

The surveillance system in Madagascar employed immediate notification of unusual event such as a time-space clustering of cases [2]. Health facilities used a standardized form to report their monthly activities, especially the number of outpatient visits for certain diseases and syndromes. To strengthen the Malagasy surveillance system, a sentinel surveillance network with daily reports of febrile syndromes sent by SMS (Short Message System) was made operational in Madagascar in 2007 [3]. All SMS from 34 sentinel health centers distributed nationwide are pooled in a database. This sentinel system has demonstrated its efficacy in Madagascar by detecting the spatiotemporal evolution of the influenza pandemic in 2009 [4].

In 2011, an evaluation of the fever sentinel surveillance system showed good quality of the collected data. The average timeliness and completeness rate of the SMS data were 78\% [95\% CI: 73-82] and 97\% [95\% CI: 95-98], respectively [5]. The site-specific incidence of reported fever cases ranged from 0 to more than 100 daily cases, with an average of 4 per week.

A regular assessment of a surveillance system is recommended by the World Health Organization (WHO) to assess the quality of the generated data and to identify the system attributes and processes that need improvement [6]. Therefore in 2014-15, six health centers were randomly selected from the sentinel network to be included in an investigation of the sensitivity of the fever sentinel surveillance system to reliably detect trends in febrile illnesses and malaria in Madagascar.

\section{Materials and Methods}

\section{Study design}

A capture-recapture approach was used to compare febrile patients presenting in sentinel health centers with febrile subjects actively detected in their respective catchment areas. The "capture" source of febrile illnesses was the outpatient registry from sentinel health centers, with identification of all registered patients presenting at least one fever episode (axillary temperature $\geq 37.5^{\circ} \mathrm{C}$ ) within the study 
week. The "recapture" source of febrile illnesses was the population census in the catchment area of study health centers, with identification of all new febrile cases through an active screening in the same study week. The catchment area population for the recapture population was defined as all individuals residing within a $5 \mathrm{~km}$ radius of the health center. Two adjacent neighbourhoods beyond $5 \mathrm{~km}$ from the health center were added to detect a possible effect of distance on the sensitivity. One neighbourhood located more than $5 \mathrm{~km}$ from the health center was randomly selected, and a second neighbourhood in the diametrically opposite side (north-south for example). Only febrile episodes that occurred during the one-week study period and within the study area were included.

\section{Study location}

Six sentinel sites were selected for the study and stratified in three strata of two health centers according to the average number of weekly reported fever cases since 2007:

- Stratum 1: Two health centers were randomly selected from the 19 health centers reporting an average of fewer than 4 fevers per week.

- Stratum 2: Two health centers were randomly selected from the 11 health centers reporting an average between 4 and 9 fevers per week.

- Stratum 3: Two health centers were randomly selected from the four health centers reporting an average above than 9 fevers per week.

\section{Data collection}

The data collection from the capture source was initiated during the passive screening in health centers. The passive screening was not influenced by the investigator team. A standardized questionnaire was administered to each patient presenting with fever to collect his or her demographics, clinical information as well as the main reason for their consultation. A "capture card" was then distributed with the following information: age, patient number, duration of the fever and neighbourhood of residence.

The recapture component in each catchment area used the same questionnaire with an additional section to identify the reasons for not attending a health center during the week-end after passive screening. A population census was carried out with home visits (a second visit if people were out at time of first visit), while geographical locations of all fever cases were recorded. The "capture cards" distributed during the recapture active screening allowed investigators to match cases common to both sources.

Acute malaria was defined as a fever syndrome with a positive malaria rapid diagnostic test. Influenza-like illness (ILI) was defined as a fever with a cough and evolution less than 7 days. Dengue-like syndrome (DLS) or arbovirosis was defined as a fever without respiratory symptoms and at least two of the following symptoms: headache, arthralgia, myalgia, skin rash, retro-orbital pain and hemorrhagic syndrome. Febrile diarrhoea was defined as a fever with 3 or more watery stools during the previous 24 hours.

\section{Statistical analysis}

All data were entered in an MS Access ${ }^{\circledR}$ database at the Epidemiology Unit of the Institute Pasteur of Madagascar. Data anonymization was performed by allocating a unique ID number to each patient. The data analysis was performed with $\mathrm{R}$ version 2.7.0 software and Stata ${ }^{\bullet}$ (version 13, Stata Corp LP, College Station, TX,
USA). A socio-demographic description of persons with fevers was followed by a time, place and person analysis. Chi-square tests and $t$ tests were deemed statistically significant whenever a $\mathrm{p}$-value was below 0.05 .

Febrile subjects identified during the same study week were recorded from both capture (registry=A) and recapture (catchment area $=\mathrm{B}$ ) sources. Using cross-tabulation of the data from both sources, a contingency table was established to display the number of fever cases which were not reported in health centers $[7,8]$. The total number of cases in the population $\mathrm{N}$ was estimated using the number of cases from each source (NA and $\mathrm{NB}$ ) and the number of common cases $\mathrm{XAB}$, according to the following formula: $\mathrm{N}=\frac{\mathrm{N}_{\mathrm{A}} \times \mathrm{N}_{\mathrm{B}}}{\mathrm{X}_{\mathrm{AB}}}$. Under the two independent sources hypothesis, the values of Sekar and Demming allowed an estimation of the number cases that were not identified by any of the sources (X0), the variance of $\mathrm{N}(\operatorname{Var} \mathrm{N})$, and the $95 \%$ confidence interval.Var $(\mathrm{N})=\frac{\mathrm{N}_{\mathrm{A}} \mathrm{N}_{\mathrm{B}} \mathrm{X}_{\mathrm{A}} \mathrm{X}_{B}}{\mathrm{X}_{\mathrm{AB}}{ }^{3}}$

$$
C I_{95 \%}(N)=N \pm 1,96 \sqrt{\operatorname{Var}(N)}
$$

And

$X_{0=}{ }^{N-}\left(X_{A B}+X_{A}+X_{B}\right)$

$X_{0}=\frac{X_{A} X_{B}}{X_{A B}}$

The sensitivity for the system was calculated as follows:

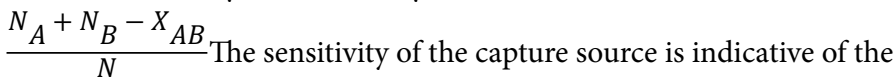
sensitivity of the fever sentinel sites to detect a disease under surveillance, whether a febrile illness or a malaria case $[9,10]$. The sensitivity of the fever cases was measured based on the estimated number of cases [11].

Six conditions are required to apply the capture-recapture method: (i) all identified cases were true cases, (ii) all identified cases occurred during the same period and in the precise geographical area studied, (iii) the study population was closed, (iv) all true duplicates and only true duplicates were identified, (v) there was homogeneity for the catchment cases and (vi) the two sources were independent [12].

\section{Ethics committee}

The protocol for this study was submitted for review to the National Ethics Committee at the Malagasy MoH. It was approved as a noninterventional research study. This study was classified as a public health practice and did not required human subject research review beyond center for Global Health.

\section{Results}

Six randomly selected health centers were investigated between August 2014 and March 2015. The first stratum included Taolagnaro and Ambositra (sites with lower consultation activity), the second included Tsiroanomandidy and Ambato Boeny (medium activity), and the third included Maevatanana and Toamasina (higher activity). Figure 1 show the sentinel surveillance network categorized by weekly incidence of febrile illnesses. The capture phase (one week of passive screening in health centers) identified 1,164 registered outpatients, of 
Citation: Randriamampionona L, Randrianasolo L, Randremanana RV, Ramarokoto C, Ramarokoto T, et al. (2018) Sensitivity of Fever Sentinel Surveillance System to Survey Malaria Trends in Madagascar, 2014-2015. J Trop Dis 6: 280. doi:10.4172/2329-891X.1000280

Page 3 of 6

which $23.2 \%(270 / 1164)$ presented fever during the study week. The recapture phase (same week active screening in catchment area) screened 149,855 persons in 79 neighbourhoods, of which $3.7 \%$ (5546/149855) had fever. Hence, a total of 5,816 febrile subjects were identified in both sources.

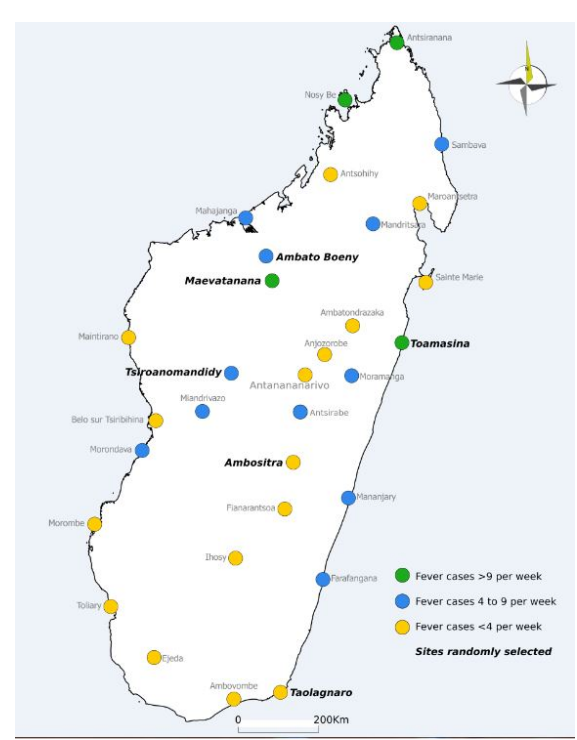

Figure 1: Geographical location of Fever sentinel sites, 2014-2015, Madagascar.

However, 2,757 febrile subjects were excluded from the capturerecapture analysis: 113/270 (41.9\%) from the capture population and $2644 / 5546$ (47.7\%) from the recapture population. The main causes of exclusion were: fever episodes ending before or beginning after the study period (75.6\%-2083/2757), living outside the defined study area (23.2\%-641/2757) and refusing to participate (1.2\%-33/2757). Of the 3,059 (5816-2757) eligible febrile subjects, 119 were common to both sources. Duplicate observations were removed and the capturerecapture analysis therefore included 2,940 febrile subjects (3059-119) (Table 1).

\section{Epidemiological description}

The overall sex ratio (male/female) of febrile subjects was 0.76 (1269/1671), and the median age was 14 years [IQR: $4-33$ years]. $57 \%$ of individuals or their parents completed secondary level education or higher (1677/2940). The median fever duration was 9 days (IQR: 70-13) with an average body temperature of $38.1^{\circ} \mathrm{C}$ [95\% CI: 37.9-38.2]. Main febrile illnesses were malaria confirm by rapid diagnostic test (0.5\%-15/2940), febrile diarrhoea (4.8\%-140/2940), influenza like illness (37.6\%-1104/2940) and other causes (57.2\%-1681/2940).

In the capture population (health centers), 157 febrile patients were included with a sex ratio (male/female) of $1.04(80 / 77)$, and their median age was 6 years [IQR: 2-21 years]. Age below 6 years and acuteness of fever were the most common reasons for consulting.

In the recapture population (community active screening), 2,783 febrile individuals were included with a sex ratio (male/female) of 0.75 (1189/1594) and a median age of 14 years [IQR: 4-4 years]. Based on census data, $4.0 \%(119 / 2940)$ of the individuals had received the study capture card.

There was a statistically significant difference between sources in the median age $(\mathrm{p}<0.001)$ and duration of fever $(\mathrm{p}<0.001,2$ days in health center versus 10 days in community). The number of associated symptoms or clinical signs, educational level of the individual/parents and gender were not determining factors for visiting the health centers.

\begin{tabular}{|c|c|c|c|c|c|c|c|c|c|c|}
\hline \multirow{3}{*}{$\begin{array}{l}\text { Stratu } \\
\text { m }\end{array}$} & \multirow{3}{*}{$\begin{array}{l}\text { Study } \\
\text { location }\end{array}$} & \multirow{3}{*}{$\begin{array}{l}\text { Field } \\
\text { investigation } \\
\text { period }\end{array}$} & \multicolumn{3}{|c|}{ Passive screening (Health Center) } & \multicolumn{4}{|c|}{ Active Screening (Population) } & \multirow{3}{*}{$\begin{array}{l}\text { Fever } \\
\text { Cases } \\
\text { identifi } \\
\text { ed by } 2 \\
\text { source } \\
\text { s } \\
\text { (matchi } \\
\text { ng rate } \\
\text { in \%) }\end{array}$} \\
\hline & & & \multicolumn{2}{|c|}{ Cases identified $(n=270)$} & \multirow[t]{2}{*}{$\begin{array}{l}\text { Outpatient } \\
\text { visits }\end{array}$} & \multicolumn{3}{|c|}{ Cases Identified $(n=5,546)$} & \multirow[t]{2}{*}{$\begin{array}{l}\text { Population } \\
\text { census }\end{array}$} & \\
\hline & & & $\begin{array}{l}\text { Included } \\
\text { cases }\end{array}$ & $\begin{array}{l}\text { Excluded For outside } \\
\text { study area }\end{array}$ & & $\begin{array}{l}\text { Included } \\
\text { cases }\end{array}$ & $\begin{array}{l}\text { Included cases } \\
\text { adjusted }^{*}\end{array}$ & $\begin{array}{l}\text { Excluded } \\
\text { cases }\end{array}$ & & \\
\hline 1 & Taolagnaro & $\begin{array}{l}2014 / 09 / 01-201 \\
4 / 09 / 06\end{array}$ & 13 & 0 & 75 & 501 & 488 & 2,013 & 45,879 & $\begin{array}{l}13 \\
(2.6 \%)\end{array}$ \\
\hline 1 & Ambositra & $\begin{array}{l}2014 / 12 / 08-201 \\
4 / 12 / 12\end{array}$ & 10 & 11 & 110 & 10 & 0 & 31 & 8,978 & $\begin{array}{l}10 \\
(100 \%)\end{array}$ \\
\hline 2 & $\begin{array}{l}\text { Ambato } \\
\text { Boeny }\end{array}$ & $\begin{array}{l}2014 / 11 / 24-201 \\
4 / 11 / 28\end{array}$ & 27 & 1 & 209 & 32 & 9 & 16 & 11,929 & $\begin{array}{l}23 \\
(71.9 \%)\end{array}$ \\
\hline 2 & $\begin{array}{l}\text { Tsiroanoman } \\
\text { didy }\end{array}$ & $\begin{array}{l}2014 / 11 / 17-201 \\
4 / 11 / 22\end{array}$ & 27 & 6 & 123 & 356 & 329 & 240 & 34,591 & $\begin{array}{l}27 \\
(7.6 \%)\end{array}$ \\
\hline 3 & $\begin{array}{l}\text { Maevatanan } \\
\text { a }\end{array}$ & $\begin{array}{l}2014 / 08 / 04-201 \\
4 / 08 / 08\end{array}$ & 63 & 58 & 346 & 119 & 83 & 75 & 13,736 & $\begin{array}{l}36 \\
(30.3 \%)\end{array}$ \\
\hline
\end{tabular}


Citation: Randriamampionona L, Randrianasolo L, Randremanana RV, Ramarokoto C, Ramarokoto T, et al. (2018) Sensitivity of Fever Sentinel Surveillance System to Survey Malaria Trends in Madagascar, 2014-2015. J Trop Dis 6: 280. doi:10.4172/2329-891X.1000280

Page 4 of 6

\begin{tabular}{|l|l|l|l|l|l|l|l|l|l|l|}
\hline 3 & Toamasina & $\begin{array}{l}2015 / 03 / 16-201 \\
5 / 03 / 20\end{array}$ & 17 & 37 & 301 & 1,884 & 1,874 & 269 & 34,742 & $\begin{array}{l}10 \\
(0.5 \%)\end{array}$ \\
\hline & Total & $\mathbf{1 5 7}$ & $\mathbf{1 1 3}$ & $\mathbf{1 1 6 4}$ & $\mathbf{2 9 0 2}$ & $\mathbf{2 7 8 3}$ & $\mathbf{2 6 4 4}$ & $\mathbf{1 4 9 , 8 5 5}$ & $\begin{array}{l}\mathbf{1 1 9} \\
\mathbf{( 4 . 1 \% )}\end{array}$ \\
\hline
\end{tabular}

Table 1: Fever cases distribution by health centers during the active and passive screening, 2014-2015.

\section{Reasons for attendance and non-attendance}

The main reasons for attendance among the 157 febrile illnesses consulting the health centers were point of care satisfaction (43.5\%), good healthcare provider-patient relationship (20.3\%), other trip purpose $(8.5 \%)$, proximity of the health center $(7.3 \%)$ and financial capacity $(6.1 \%)$. At health center, the work flow to provide care management was clearly marked, the need for prescribed medication/s and clinical signs perceived as severe were cited but represented less than $5 \%$ of the reported reasons for attendance.

The main reasons for not consulting the sentinel sites among the 2,783 febrile subjects in neighbouring communities were: other preferences for consultation (35.6\%), poverty (25.7\%), and clinical symptoms not perceived as severe $(10.5 \%)$, dissatisfaction in previous visits $(6.5 \%)$ and remoteness of the health center (4.8\%). Other reasons such as unsatisfactory patient-provider rapport and the several appointments with the health care provider were cited but less than $5 \%$.

\section{Estimation of the number of fever cases}

We supposed that the six conditions to estimate cases not identified by either sources (Sekar and Demming) were verified:

1. In passive screening, the case definition used for the fever syndrome was an axillary temperature $\geq 37.5^{\circ} \mathrm{C}$. Therefore, all identified cases were true cases.

2. The exclusion criteria were individuals living outside the defined study area and individuals who had a fever syndrome that ended before or began after the inclusion period in the health centers. All identified cases occurred during the same study period and in the same geographical area.

3. The study population included only those who were present or close to the health centers during the inclusion period.

4. The capture card was delivered to the health centers and filled out during the active screening for recapture. Therefore, all identified common cases were real.

5. The two sources were independent: the first from the health centers and the second from the population census.

6. All cases in the study population had the same probability of being identified at the health centers. No statistically significant variations in gender, diagnosis or level of education were demonstrated.

As capture-recapture method with two sources, 3,829 [95\% CI: 3498-4160] were the estimated cases and minus by 2,940 cases observed during the field study, 889 febrile cases were probably unidentified by the sentinel sites. Therefore $76.8 \%$ (2940/3829) was the sample coverage of the sentinel sites (Figure 2).

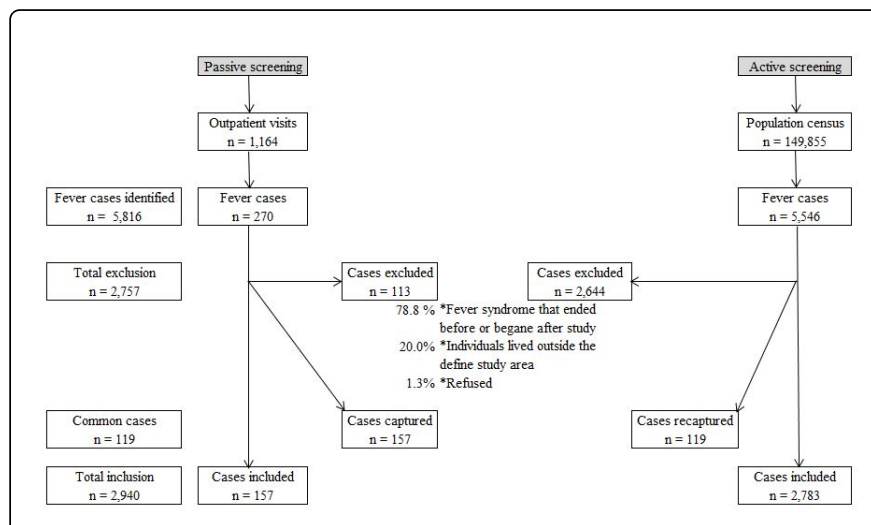

Figure 2: Inclusion flow chart for fever cases capture-recapture, 2014-2015.

\section{Sensitivity of fever and diseases surveillance in sentinel sites}

The sensitivity to document febrile illnesses in the sentinel health centers was $4.1 \%$ (157/3829) [95\% CI: 3.8-4.5]. In each of the three strata, the estimated febrile cases were 511, 419 and 3,484, which corresponded to a sensitivity of $4.5 \%$ [95\% CI: 3.0-6.7] in lower attendance centers, $12.9 \%$ [95\% CI: 12.0-13.9] in medium attendance centers and 2.3\% [95\% CI: 1.9-2.8] in higher attendance centers. A comparison of sensitivity between strata showed a statistically significant difference $(\mathrm{p}<0.001)$ (Table 2).

\begin{tabular}{|l|l|l|l|l|l|l|}
\hline & $\begin{array}{l}\text { Mean } \\
\text { of } \\
\text { weekly } \\
\text { fever } \\
\text { cases } \\
\text { declare } \\
\text { d }\end{array}$ & $\begin{array}{l}\text { Fever } \\
\text { cases } \\
\text { identifie } \\
\text { d by } \\
\text { health } \\
\text { center }\end{array}$ & $\begin{array}{l}\text { Estimatio } \\
\text { n of fever } \\
\text { cases into } \\
\text { the } \\
\text { catchment } \\
\text { area }\end{array}$ & [95\% Cl] & $\begin{array}{l}\text { Sensiti } \\
\text { vity }\end{array}$ & $\begin{array}{l}{[95 \%} \\
\text { CI] }\end{array}$ \\
\hline $\begin{array}{l}\text { Stratum 1 } \\
\text { (Taolagnaro, } \\
\text { Ambositra) }\end{array}$ & $<4$ & 23 & 511 & $\begin{array}{l}{[343.3-76} \\
6.7]\end{array}$ & $4.50 \%$ & {$[3.0-6.7$} \\
\hline $\begin{array}{l}\text { Stratum } \\
\text { (Tsiroanoma } \\
\text { ndidy, } \\
\text { Ambato } \\
\text { Boeny) }\end{array}$ & $4-9$ & 54 & 419 & $\begin{array}{l}{[389.5-44} \\
8.5]\end{array}$ & $12.90 \%$ & {$[12.0-1$} \\
\hline $\begin{array}{l}\text { Stratum 3 } \\
\text { (Maevatanan } \\
\text { a, } \\
\text { Toamasina) }\end{array}$ & $>9$ & 80 & 3,484 & $\begin{array}{l}\text { [2835.3-4 } \\
132.7]\end{array}$ & $2.30 \%$ & {$[1.9-2.8$} \\
\hline
\end{tabular}


Page 5 of 6

\begin{tabular}{|l|l|l|l|l|l|}
\hline All six health centers & 157 & 3,829 & $\begin{array}{l}{[3497.6-4} \\
160.4]\end{array}$ & $4.10 \%$ & {$[3.8-4.5$} \\
\hline
\end{tabular}

Table 2: Estimation of fever syndromes in the catchment area by stratum, 2014-2015.

Unidentified malaria cases were estimated at 2 out of 17 [95\% CI: 7-27] acute malaria cases in both sources. Of notice, $88 \%(15 / 17)$ of acute malaria cases were identified in both sources. The sensitivity of identifying cases of acute malaria in the sentinel health centers was therefore $70 \%(12 / 17)$. For ILI and febrile diarrhea, the sensitivity to identify cases was $2.9 \%$ and $8.8 \%$, respectively.

\section{Space-time distribution of diseases under surveillance in the population}

The weekly prevalence of fever syndrome was estimated at $2.6 \%$ $(3829 / 149855)$ in the catchment area population. The weekly cumulative incidence was $0.01 \%(15 / 149855)$ for malaria, $0.09 \%$ (140/149855) for diarrhoea and $0.7 \%(1104 / 149855)$ for ILI. When extrapolating, the yearly cumulative incidence of acute malaria, diarrhoea and ILI were respectively $0.5 \%, 4.7 \%$ and $36.4 \%$.

The disease distributions varied with study locations. Comparison of diseases incidence such as acute malaria, ILI and febrile diarrhoea showed significant differences by health center $(\mathrm{p}<0.002)$. In Maevatanana and Ambato Boeny, with a sub-humid bio climate, the yearly malaria incidence was above $5 \%$, with specific incidences of $6.2 \%$ and $5.6 \%$, respectively. For the other bio climates, the malaria incidence was below $1 \%$. For ILI and febrile diarrhoea, the weekly morbidity ranged from 10 to $49 \%$ and 0 to $10 \%$, respectively (Figure 3 ). No suspected cases of arbovirosis (dengue like syndrome) were diagnosed during the study period.
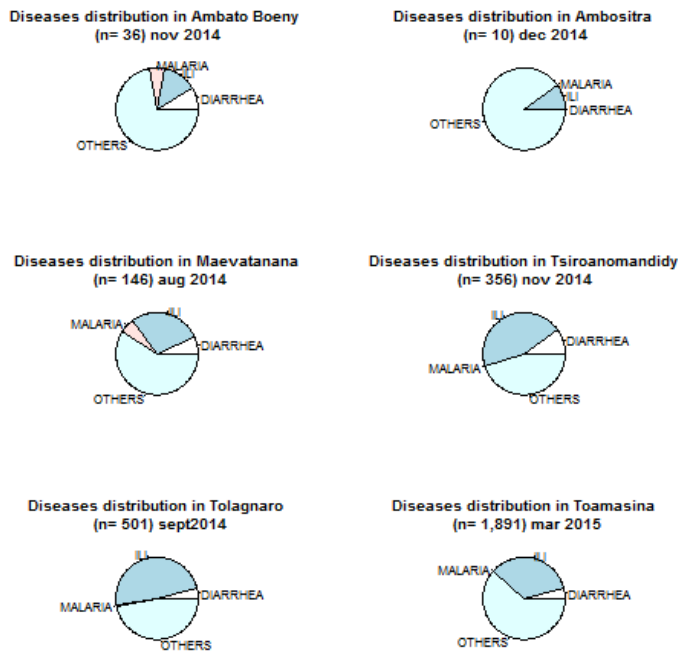

Figure 3: Diseases distribution by study location $(n=2,940)$, 2014-2015.

\section{Discussion}

The objective of this study was to evaluate the sensitivity of fever sentinel surveillance system to detect febrile illnesses in Madagascar. A capture-recapture method compared febrile illnesses presenting at the sentinel site health facilities with all febrile illnesses in their respective catchment areas was used. This method is primarily used to estimate the completeness of notifications and to estimate the size of animal populations in a geographical location $[13,14]$. The proportion of fever case captured by health centers with medium attendance was threefold higher than the other two strata. The fever sentinel surveillance system in Madagascar had a low sensitivity to detect fevers. This result may be linked to the weakness of the health system in Madagascar, as evidenced by the low outpatient department attendance $(35.7 \%$ in 2013) $[15,16]$ and poverty. In addition, individuals end up paying for healthcare and the average cost for one visit with treatment increased threefold from 2005 to 2010 (from \$1.80 to \$5.60). Health insurance or pooling healthcare exists, but less than $1 \%$ of the Malagasy population benefits from such services. Approximately $40 \%$ of the Malagasy population lives in remote rural areas which are further than $5 \mathrm{~km}$ from a health facility and tend to self-medicate or neglect fever syndromes $[15,17]$.

Breman et al. cited that more than $80 \%$ of febrile persons do not utilize the formal health infrastructure [18]. Using this information, the sample coverage in this study appears to be high compared to disease surveillance systems in Africa. For example, measles and acute flaccid paralysis surveillance system in Benin and Ivory Coast which showed a sample coverage of $28 \%$ and $40 \%$, respectively $[19,20]$.

The distribution of the diseases under surveillance varied significantly between the health centers $(\mathrm{p}<0.001)$ as a result of the different bio climates. The incidence of malaria was approximately $5.0 \%$ in the sub-humid bio climate, specifically in Maevatanana and Ambato Boeny, with respective incidences of $6.2 \%$ and $5.6 \%$. The incidence was $<1 \%$ in the other bio climates (per-humid and humid). These results confirmed the malaria trends reported by Kesteman et al. [21].

\section{Limitations}

The first limitation of this study was the low number of malaria cases, detected. And the second limitation was the organization of the field investigation. The passive screening was conducted during business hours in health centers while the active screening was performed after business hours. However, the population census, the geolocation of households and revisiting in case of absent household members helped unsure the completeness of the recapture survey. Finally, the high rate of exclusion and the reliance on measured temperature at a single encounter maybe limited this study.

\section{Conclusion}

This study allowed evaluating the sensitivity of a fever sentinel surveillance system in Madagascar. The sensitivity for capturing fever cases by health centers was low at $4.1 \%$, but much higher at $70.0 \%$ for acute malaria. These results will serve as a baseline for future evaluative research of the fever sentinel surveillance system in Madagascar.

\section{Acknowledgments}

We are grateful to all those who participated in this study, including, in particular, Arthur Randrianantenaina, Jean Marius 
Citation: Randriamampionona L, Randrianasolo L, Randremanana RV, Ramarokoto C, Ramarokoto T, et al. (2018) Sensitivity of Fever Sentinel Surveillance System to Survey Malaria Trends in Madagascar, 2014-2015. J Trop Dis 6: 280. doi:10.4172/2329-891X.1000280

Page 6 of 6

Rakotondramanga, Andritsilavo Jean Narivelo and Lucie Randrianarivony from Epidemiology and clinical research unit at the Institut Pasteur de Madagascar, for their technical and administrative assistance. We are also thank Meltine Razanakiniaina, Tsimaholy Zanah Rahasivelo, Mamisoa Razafitsiferana, Fanjanirina Rasendranoronaivoson, Hery Rakotoniaina and Daniel Randriamiravonjy, head of the fever sentinel sites from the Ministry of Public Health for their cooperation during the field investigation. We fill with gratitude to Barbara Marston, Peter McElroy, Ryan Wiegand and S.P. Kachur, Malaria Branch, US Centers for Disease Control and Prevention, for their critical revision of this manuscript.

\section{References}

1. World Health Organization (WHO) (2007) Global plan to combat neglected tropical diseases 2008-2015.

2. World Health Organization (WHO) and Centers of disease control and prevention (2001) technical guidelines for integrated disease surveillance and response in the African Region.

3. Randrianasolo L, Raoelina Y, Ratsitorahina M, Ravolomanana L, Andriamandimby S, et al. (2010) Sentinel surveillance system for early outbreak detection in Madagascar. BMC Public Health 10: 31.

4. Rajatonirina S, Heraud JM, Orelle A, Randrianasolo L, Razanajatovo N, et al. (2012) The spread of Influenza A(H1N1)pdm09 Virus in Madagascar described by a sentinel surveillance network. PLoS ONE; 7: e37067.

5. Randrianasolo L, Ravololomanana L, Rakotoarivony C, Rasoamanarivo H, Ramarosandratana B, et al. (2013) Evaluation of the fever sentinel surveillance system in Madagascar.

6. World Health Organization (WHO) (1997) Protocol for evaluation of epidemiological surveillance systems.

7. Sekar C, Deming EW, (1949) On a method of estimating birth and death rates and extent of registration. J Am Stat Assoc 44: 101-115.

8. Chao A, Pan HY, Chiang SC (2008) The Petersen-Lincoln estimator and its extension to estimate the size of a shared population. Biometrical Journal 50: 957-970.

9. Coureau G (2010) Master of sciences, technologies and health, option public health, 2009-2010: The capture-recapture methods. Bordeaux : Université Victor Segalen Bordeaux 2, ISPED.
10. Spaccaferri G, Cazein F, Lièvre L, Geffard S, Gallay A, et al. (2010) Estimation of completeness of AIDS surveillance with capture-recapture method, France 2004-2006. Institut de Veille Sanitaire.

11. Gallay A, Nardone A, Vaillant V, Desenclos JC (2002) The capturerecapture applied to epidemiology : principles, limits and application. Rev Epidemiol Sante Publique 50 : 219-232.

12. Chao A, Tsay PK, Lin SH, Shau WY, Chao DY (2001) The applications of capture-recapture models to epidemiological data. Stat Med 330: 3123-3157.

13. Naradone A, Decludt B, Jarraud S, Etienne J, Hubert B, et al. (2003) Repeat capture-recapture studies as part of the evaluation of the surveillance of Legionnaires' disease in France. Epidemiol Infect 131: 647-654.

14. Van Hest NAH, Smit F, Verhave JP (2002) Underreporting of malaria incidence in the Netherland: results from a capture-recapture study. Epidemiol Infect 129: 371-377.

15. Ministry of Health in Madagascar (2015) Health sector development plan 2015-2019.

16. Olds P (2006) Access to medecines in Madagascar: The state, the drugstore and the users. Fort-Dauphin, Madagascar. Independent Study Project (ISP) Collection. Paper 259.

17. Mattern C, Pourette D, Raboanary E, Kestman T, Piola P, et al. (2016) "Tazomoka is Not a Problem". Local perspectives on malaria, fever case management and bed net use in Madagascar. PLose ONE 11: e0151068.

18. Breman JG (2001) The ears of hippopotamus: manifestations, determinants and estimates of the malaria burden. Am J Trop Med Hyg 64: 1-11.

19. Rey JL, Trolet C, Soro B, Cunin P, Merouze F (1991) Difficulties in the epidemiological surveillance of measles in Africa : the Ivory Coast as an example. Ann Belgian Soc Trop Med 71: 115-21.

20. Saizonou J, Drabo M, Sossa C, Sodjinou V, Glélé Y, et al. (2014) Performance of measles epidemiological surveillance system in Benin in the context of its elimination. Medicine of Black Africa. 61: 123-132.

21. Kesteman T, Randrianarivelojosia M, Mattern C, Raboanary E, Pourette D, et al. (2014) Nationwide evaluation of malaria infections, morbidity, mortality, and coverage of malaria control interventions in Madagascar. Malar J 13: 465. 